\title{
Algebra of Real Functions: Classification of Functions, Fictitious and Essential Functions
}

\author{
Maydim Malkov \\ Department of Mathematics, Russian Research Center for Artificial Intelligence, Moscow, Russia \\ Email address: \\ mamalkov@gmail.com \\ To cite this article: \\ Maydim Malkov. Algebra of Real Functions: Classification of Functions, Fictitious and Essential Functions. Pure and Applied Mathematics \\ Journal. Vol. 8, No. 4, 2019, pp. 72-76. doi: 10.11648/j.pamj.20190804.11
}

Received: July 25, 2019; Accepted: August 15, 2019; Published: September 3, 2019

\begin{abstract}
Real numbers are divided into fictitious (non-computable) and essential (computable). Fictitious numbers do not have numerical values, essential numbers have algorithms for constructing these numbers with any exactness. The set of fictitious numbers is continual, the set of essential numbers is countable. Functions are also divided into fictitious, defined over the set of fictitious numbers, and essential, defined over the set of essential numbers. Essential functions have an algorithm for calculating any value with any exactness. All functions of applied mathematics and some functions of abstract mathematics are essential The set these functions is countable. The four upper levels of classification of real functions are constructed. This classification uses superpositions of functions and diagonal sets borrowed from the algebra of finite-valued functions.
\end{abstract}

Keywords: Algebra of Real Functions, Algebra of Superpositions, Computable Real Functions, Computable Real Numbers

\section{Introduction}

There is a vast number of papers devoted to classification of real functions. This is due to the lack of a satisfactory classification of real functions.

All these classifications ignore the classification of functions preceding real ones. Using this classification, many problems of real function classification would be solved.

The classification of real functions must begin at bottom, at the classification of Boolean functions, finite valued functions, and countably valued functions.

A complete classification of Boolean functions was constructed by E. L. Post [1]. Some addition was given in the study [2]. The main means of constructing this classification are superpositions (compositions) and diagonal sets. Superpositions are variables permutation, identification of variables and substitution of functions instead of variables of another function. Diagonals are functions with the same variable values. There are 4 diagonal sets of Boolean functions, they are the sets $\alpha, \beta, \gamma, \delta$. There are countable diagonal sets in real function algebra.

The classification of finite-valued functions was constructed in $[3,4]$. The main difficulty of this classification is the continuity of the set of subalgebras in algebra of these functions [5]. The classification of subalgebras in this algebra is also a classification of functions. It turned out that there are fictitious subalgebras to be useless for classification of functions [6]. The set of these subalgebras is continual, and the set of essential subalgebras is countable.

Nevertheless, it is not possible to construct a complete classification even of three-valued functions due to an incomparably larger number of essential subalgebras with respect to subalgebras of two-valued functions.

Many authors tried to solve the problem of classifying finite valued functions using stronger closure operations with respect to superpositions $[7,8]$. These operations reduce the set of subalgebras, which allows to construct a complete classification. But the use of these classifications is insignificant. Instead of these closure operations, one can use superpositions, but restrict oneself to a few upper levels of classification.

Superpositions and diagonal sets are applicable in the algebra of real numbers. Using these superpositions and diagonal sets, 4 upper levels of classification of real functions are constructed in this article.

Classification of real numbers will receive a powerful push by applicating methods of classification of finite-valued and countable-valued functions. 


\section{Basic Definitions}

\subsection{Definition of Algebra of Real Functions}

A. I. Malcev gave a definition of algebras of any functions. This definition is given below for real functions.

Definition. Algebra of real functions $\mathrm{P}_{\mathbb{R}}$ is

$$
\mathrm{P}_{\mathbb{R}}=\left(P_{\mathbb{R}} ; \Omega\right)
$$

where $P_{\mathbb{R}}$ is the carrier of algebra, it contains all functions over the set of real numbers $\mathbb{R}$. The set $\Omega$ contains basic operations of algebra. These are operations of superpositions (compositions):

1. cyclic permutation of variables, at which the first variable becomes the last, respectively numbering of variables is changed (variables are numbered from one to $n$ );

2. permutation of the first and last variables; this and previous operations perform any variable permutation;

3. identification (equality) of the first and last variables;

4. replacing the first variable of a function with another function.

A. I. Malcev called this algebra Post algebra. The designation $P_{\mathbb{R}}$ is given in honor of E. L. Post.

A subalgebra of this algebra is an algebra whose carrier is a set of functions closed by superpositions, and whose basic operations are superpositions.

Further, subalgebras of algebra $\mathrm{P}_{\mathbf{R}}$ are called algebras.

Since all algebras contain the same set of basic operations, it is generally accepted to identify algebra and its carrier. This simplifies the formulation of statements. In particular, the intersection of two algebras is an algebra whose carrier is the intersection of the carriers of these two algebras. The union of two algebras (union of carriers) may not be an algebra.

\subsection{Essential and Fictitious Numbers}

Real functions are defined over the set of real numbers.

In following, real functions are called functions, and real numbers are called numbers.

The numbers are divided into essential and fictitious. Essential numbers are numerical, i.e. are computable. The set of these numbers is countable. Numbers that do not have a numerical value are fictitious. The set of fictitious numbers is continual (the set of fictitious objects exists in almost every theory, and this set is always incomparably larger than the set of essential objects).

Fictitious numbers have no numerical value. These numbers are useless.

Definition. A number is called essential (computable) if there is an algorithm for calculating this number with any exactness. The remaining numbers are called fictitious (not computable).

The set of fictitious numbers is continual. The set of essential numbers is countable, as the set of algorithms is countable.

The classification of numbers contains two classes: essential (computable) numbers and fictitious numbers. The class of computable numbers also contains two classes: rational and irrational numbers. The class of irrational numbers contains the class of algebraic numbers and the class of transcendental numbers. This completes classification of numbers.

The set of computable numbers is denoted by $\mathrm{R}$.

Complex numbers are also essential and fictitious.

\subsection{Essential and Fictitious Functions}

The set of all functions is hyper continual. But the set of essential (computable) functions is countable.

Definition. A function is called computable if it is computed with any exactness for any computable numbers, for which this function is not infinite.

All functions existing in applied mathematics are computable. And some functions of abstract mathematics are computable. For example, Dirichlet function is computable.

\section{Classification of Functions}

\subsection{About Classification}

The main problem of any theory is classification of its objects. In $\mathrm{P}_{\mathbb{R}}$ objects are functions, and the first step of the classification contains two classes: computable and non-computable functions.

Classification of non-computable functions is useless. Further the classification is given for computable functions, non-computable functions are not used and all functions are considered computable.

The set of functions forms the algebra $P_{R}$. This algebra contains fictitious functions (functions with fictitious variables), but without them the algebra becomes partial, since set of essential functions closed by superpositions contains fictitious functions. In the following, only closed sets of functions are investigated. Each closed set is a subalgebra of the algebra $P_{R}$. Further, subalgebras are called algebras.

Not all algebras implement the classification of functions. Only algebras generated by a one-element basis implement the classification of functions. The remaining algebras are fictitious, since they are useless for classifying functions.

Indeed, each function generates some algebra. The set of functions generating the same algebra forms a class of bases, and these classes do not overlap. Each function belongs to only one class, i.e. classes of one-element bases and the algebras generated by them really to classify functions. Algebras generated only by multi-element bases are useless for classifying functions. In the following, only algebras generated by one-element bases are investigated.

\subsection{Baire Classification}

The second level of classification contains sets of discontinuous functions.

The class 0 of this classification is a set of continuous functions. The class 1 a set of discontinuous functions which have points to be represented as the limit of a convergent sequence of continuous functions. Any other class does not contain functions of previous classes, but functions of this class have points to be represented as the limit of a convergent sequence of continuous functions of the previous class. 
An example is Dirichlet function

$$
f(x)=\lim [\cos n ! \pi x]^{2 m} \text { at } n, m \rightarrow \infty,
$$

which belongs to class 2 . And there are functions that do not belong to any of these classes. They form one more class, but functions of this class are not computable.

\subsection{Maximal Algebras}

The next level of classification of continuous functions contains sets of maximal algebras, i.e. algebras contained only in Baire class 0 . The set of these algebras is countable, since the set of computable functions generating these algebras is countable.

Many results of papers devoted to maximal algebras of finite-valued functions apply to functions too.

In particular, S. V. Yablonsky and I. Rosenberg established 6 sets of maximal algebras of finite-valued functions $[9,10]$.

These are sets of algebras: monotone functions, automorphic functions, linear functions, functions that preserve central relation, functions that preserve nontrivial equivalence relation, the union of one-place and incomplete-valued functions.

The function $f\left(x_{1}, \ldots, x_{n}\right)$ is monotone if

$$
\begin{gathered}
\left(a_{1}, \ldots, a_{n}\right) \leq\left(b_{1}, \ldots, b_{n}\right) \rightarrow f\left(a_{1}, \ldots, a_{n}\right) \\
\leq f\left(b_{1}, \ldots, b_{n}\right)
\end{gathered}
$$

where

$$
\left(a_{1}, \ldots, a_{n}\right) \leq\left(b_{1}, \ldots, b_{n}\right) \text { if } a_{i} \leq b_{i}
$$

This definition is a natural extension from the monotony of one-place functions to the monotony of multi-place functions.

In addition to the usual order of monotony, any other computable order can be used.

A function is automorphic if

$$
\begin{array}{r}
\exists s \forall\left(x_{1}, \ldots, x_{n}\right) f\left(s\left(x_{1}\right), \ldots, s\left(x_{n}\right)\right) \\
=s\left(f\left(x_{1}, \ldots, x_{n}\right)\right)
\end{array}
$$

where $s$ is a single-place all-valued function consisting of cycles of the same length $p$ ( $p$ is a prime number). The function $s$ has a cycle of length $p$ if $p$ times there is $s(s(\ldots(s(x)) \ldots))=x$ for all $x$.

Function is linear if

$$
\begin{gathered}
\exists\left(a_{0}, \ldots, a_{n}\right) \forall\left(x_{1}, \ldots, x_{n}\right) f\left(x_{1}, \ldots, x_{n}\right) \\
=a_{0}+a_{1} x_{1}+\ldots+a_{n} x_{n}
\end{gathered}
$$

The operations of addition and multiplication can be replaced by dual operations. I. e. when the addition $\oplus$ is $x_{1} \oplus x_{2}=s^{-1}\left(s\left(x_{1}\right)+s\left(x_{2}\right)\right)$, and when the multiplication of $\odot$ is $x_{1} \odot x_{2}=s^{-1}\left(s\left(x_{1}\right) \cdot s\left(x_{2}\right)\right)$, where $s$ is any one-place function.

A function preserves central relation if this relation is total reflexive, total symmetric and has a center.
A relation is total reflexive if it is reflexive for multi-variable relations, i.e. there is at least one pair of equal variables. A relation is total symmetrical if it is multi-variable and symmetrical for all variables. A relation $r$ has a center if some first variables have a fixed value, for example, $r=$ $\left(0,1, x_{3}, x_{3}, x_{5}, \ldots, x_{n}\right)$. A function preserves a nontrivial equivalence relation if this relation classifies functions, but does not generate classes, each of which has a single element, and does not generate a single class. A function incomplete-valued if it does not accept all values.

But these 6 sets of algebras have only 3 sets of essential algebras, the remaining 3 sets contain fictitious algebras [11].

G. Rosseau and P. Schofield proved that only 3 sets of maximal algebras really exist $[12,13]$. These are algebras of cellular functions, functions preserved by congruence, and automorphic functions. These authors proved that a function is complete if it does not belong to these three sets of algebras.

Function is cellular if

$$
X \subset \mathrm{R} \wedge \forall\left(x_{1}, \ldots, x_{n}\right) \in X: f\left(x_{1}, \ldots, x_{n}\right) \in X
$$

The function $f$ is preserved by congruence, if

$$
\begin{gathered}
\exists D \forall\left(x_{1}, \ldots, x_{n}, y_{1}, \ldots, y_{n}\right): x_{1} \sim y_{1} \wedge \ldots \wedge x_{n} \sim y_{n} \\
\rightarrow f\left(x_{1}, \ldots, x_{n}\right) \sim f\left(y 1<\ldots, y_{n}\right)
\end{gathered}
$$

where $D$ is a partition of $\mathrm{R}$ into disjoint sets, and $\sim$ means membership to the same of these sets. Further classification is voluminous and requires a large series of articles for its presentation. Below some tools are present for building further classification of functions.

\subsection{Diagonal Sets}

Diagonal sets are a powerful tool for classifying functions. E. L. Post used diagonal sets for classification of two-valued functions.

Definition. Let $X$ be a subset of R. Diagonal set is a set of functions $f$ with diagonals $f(x, \ldots, x)=x$ if $x \in X$, and with diagonals $f(x, \ldots, x) \neq x$ if $x \notin X$. In other words, the diagonal set preserves some elements of $R$. These elements are $x$ from $f(x, \ldots, x)=x$.

The set of preserved elements is name of a diagonal set. This set is denoted by $\mathcal{D}$. The diagonal set $\mathcal{D}=\{\}$ is called $\delta$ set. The diagonal set $\mathcal{D}=R$ is called $\alpha$ set. These names are inherited from the algebra of two-valued functions.

A function is called a function of $\mathcal{D}$, if it preserves all elements of $\mathcal{D}$. A basis is called basis of $\mathcal{D}$, if functions of this bases are of $\mathcal{D}$. An algebra is called algebra of $\mathcal{D}$, if bases of this algebra are of $\mathcal{D}$ (it will be proved below that all bases of any algebra are of the same $\mathcal{D}$ ). But this algebra may contain algebras of other $\mathcal{D}$.

An element $x \in \mathcal{D}$ is called true diagonal. For this element, $f(x, \ldots, x)=x$. An element $x \notin \mathcal{D}$ is called false diagonal. $F(x, \ldots, x) \neq x$ holds for this element. A function has true diagonal $x$ if $f(x, \ldots, x)=x$. A function has false diagonal $x$ if $f(x, \ldots, x) \neq x$.

In following, all true diagonals are called briefly diagonals, unless otherwise are specified. 
A constant $c$ is named $\{c\}$, since a constant diagonal is this constant.

Lemma. A function $f$ with diagonal $a$ generates function with diagonal $a$.

Proof. Permutation and identification of variables of a function do not change any diagonal of this function. The substitution of a function $f_{1}$ with diagonal $a$ into a function $f_{2}$ with the same diagonal is a function also with this diagonal $a$, since:

$$
f_{2}\left(f_{1}(a, \ldots, a), a, \ldots, a\right)=f_{2}(a, \ldots, a)=a
$$

But a function can generate functions of another diagonal set if this function has a false diagonal.

Lemma. A function of diagonal set $X=\left\{j_{1}, \ldots, j_{m}\right\}$ can generate only functions of diagonal set $Y \supseteq X$.

Proof. The permutation and identification operations of variables of a function do not change the diagonal set of this function. The substitution operation can change diagonal sets.

Let functions $f_{1}^{n_{1}}$ and $f_{2}^{n_{2}}$ are of the same diagonal set $X$, let

$$
\begin{aligned}
& f\left(x_{1}, \ldots, x_{n_{1}+n_{2}-1}\right) \\
& =f_{1}^{n_{1}}\left(f_{2}^{n_{2}}\left(x_{1}, \ldots, x_{n_{2}}\right), x_{n_{2}+1}, \ldots, x_{n_{1}+n_{2}-1}\right)
\end{aligned}
$$

and let this function $f$ be of diagonal set $Y$. Then

$$
f(j, \ldots, j)=j
$$

for any $j \in X$. In this case, $j \in Y$ for all $j \in X$, and $Y \supseteq X$, since $f(j, \ldots, j)$ can be $j$ for some $j \notin X$. But if $f(j, \ldots, j) \neq j$ for all $j \notin X$, then $Y=X$.

Corollary. A function of diagonal set $\alpha$ generates functions of this diagonal set only. A function of diagonal set $\delta$ can generate functions of any diagonal set.

Proof. The diagonal set $\alpha$ is denoted by $X=\mathrm{R}$, and $X$ cannot be a proper subset of any other set of numbers. The diagonal set $\delta$ is denoted by $X=\{\}$, and $X$ is a proper subset of any other set.

It follows from the lemma that a function to be not $\delta$ function does not generate $\delta$ function. But several functions of different diagonal sets can generate $\delta$ functions.

Lemma. Functions, that are not $\delta$ functions and are of different diagonal sets, can generate a $\delta$ function.

Proof. It is enough to use two functions. Let functions $f_{1}$ and $f_{2}$ be of some diagonal sets $X_{1} \neq \varnothing$ and $X_{2} \neq \emptyset$. Let

$$
X_{1} \cap X_{2}=\emptyset, f_{2}\left(x_{1}, \ldots, x_{n_{2}}\right) \in X_{2}
$$

And

$$
\forall x_{1} \in X_{2} ; \forall\left(x_{2}, \ldots, x_{n_{1}}\right) f_{1}\left(x_{1}, \ldots, x_{n_{1}}\right) \notin X_{1}
$$

Then

$$
f_{1}\left(f_{2}(x, \ldots, x), x, \ldots, x\right) \neq x
$$

i.e. the result of this substitution is a $\delta$ function.

Full functions, i.e. function generating all other functions, are only of diagonal set $\delta$.

All functions to be bases of an algebra are of the same diagonal set.

Theorem. If a function contained in a basis is of some diagonal set, then all other functions to be bases also are of this diagonal set.

Proof. If a basis is of diagonal set $\alpha$, then all bases are of this diagonal set, since basis of diagonal sets $\alpha$ generates other bases only of this diagonal set. If a basis is of diagonal set $\delta$, then this basis can generate functions of any diagonal set $X$. But bases of this $X$ do not generate functions diagonal set $\delta$, i.e. they are of another algebra. A similar result exists for functions of diagonal set other than $\alpha$ and $\delta$.

Corollary. An algebra to be of a diagonal set except $\delta$ does not contain algebra of diagonal set $\delta$.

Proof. The bases of this algebra have not $\delta$ functions and do not generate algebras of diagonal set $\delta$.

It follows from this theorem that each algebra belongs to algebras, which contain bases of this algebra. Since diagonal sets do not intersect, they form a level of classification.

Each algebra may contain algebras of other diagonal set, among which there may be diagonal set of this algebra too.

The following theorem is on diagonal sets of maximal algebras.

Theorem. All maximal algebras are of one-element diagonal sets or diagonal sets $\delta$.

Proof. Let algebra belong to a multi-element diagonal set, i.e. this algebra is not of one-element diagonal set and diagonal set $\delta$. Then this algebra is of one of one-element diagonal set algebras, if this element is of this multi-element diagonal set.

Algebras of diagonal set $\delta$ are not contained in algebras of other diagonal set. Consequently, they may be maximal. $\square$

\section{Conclusion}

The algebra of real functions is constructed and the natural classification of its subalgebras is given.

The first level of this classification contains algebras of computable and non-computable functions. Computable functions are defined over the set of computable numbers. The set of computable functions is countable. Non-computable functions are defined over the set of all real numbers. These functions practically do not exist. They are defined over a set containing numbers that do not have numerical values. The set of non-computable functions is hyper-continental.

The definition of computable numbers is given. The set of these numbers is countable.

The classification of real functions is constructed. The first level of this classification contains all the functions. The second level of this classification contains computable and non-computable functions. Further classification is constructed for computable functions, since the construction of the classification of non-computable functions is meaningless. The constructed classification of functions is at the same time a classification of essential algebras too. Essential algebras have singleton bases. All other algebras are fictitious. The third level of classification contains Baire classes. It is shown that the fourth level contains not 6 , but 3 
sets of maximal algebras of functions, since 3 sets contain fictitious algebras. Further classification of functions is not built.

All functions of applied mathematics are computable. In particular, all analytic functions are computable.

\section{References}

[1] E. L. Post, Two-valued iterative systems of mathematical logic, Princeton, Princeton Univ. Press (1941).

[2] M. A. Malkov, Logic algebra and Post algebra (theory of two-valued functions) (Russian), Moscow, Mathematical logic (2012).

[3] D. Lau, Functions algebra on finite sets, Berlin, Springer (2006).

[4] A. I. Malcev, I. A. Malcev, Iterative Post algebras, Moscow, Nauka (2012).

[5] Ju. I. Janov, A. A. Muchnik, On existence of k-valued closed classes without finite basis (Russian), Dokl. Acad. Nauk SSSR, (1), 44-46 (1959).

[6] M. A. Malkov, Classification of closed sets of functions in multi-valued logic, SOP Transactions on applied Math., (1:3), 96-105 (2014).
[7] A. V. Kuznetsov, On means for detecting of non-deductibility and inexpressibleness (Russian), in Logical conclusion, Moscow, Nauka, 5-33 (1979).

[8] S. S. Marchenkov, On FE-precomplete classes of countable logic (Russian), Discrete Mathematics, (28: 2), 51-57 (2016).

[9] S. V. Yablonsky, Functional constructions in k-valued logic (Russian), Proceedings of Mat. Institute of the USSR Academy of Sciences. V. A. Steklova, (51) 5-142 (1958).

[10] I. G. Rosenberg, Über die functionale vollständigkeit in dem mehrvertigen logiken von mehreren verändlichen auf endlichen mengen, Rozpravy Cs. Academic Ved. Ser. Math. Nat. Sci., (80) 3-93 (1970).

[11] M. Malkov, Algebra of finite-valued functions: Classification of functions and subalgebras, essential and fictitious subalgebras, Pure and Applied Math. J., (8: 2) 30-36 (2019).

[12] G. Rousseau, Completeness in finite algebras with a single operation, Proc. Amer. Math. Soc., (18), 1009-1013.

[13] P. Schofield, Independent conditions for completeness of finite algebras, J. London Math. Soc., (44) 413-423 (1969). 